\title{
A Computational Modeling for Knowledge Binding of the Unstructured Web Data
}

\author{
Patil N S, Kiran P, Preethi B
}

\begin{abstract}
The focus of this manuscript is laid towards extracting insightful data embedded into web-based information which is crucial for various academic and commercialized application requirements. The study thereby introduces a robust computational modeling by means of computing knowledge from collaborative web-based unstructured information. For this purpose, this design is simplified with Fuzzy based matching algorithm and also with a set of procedures which reduces the computational effort to a significant extent. The numerical theoretical analysis shows that the effectiveness of the formulated model. It also shows that the formulated concept outperforms the baseline modeling by almost $50 \%$ when computational performance is concerned.
\end{abstract}

Keyword: Unstructured web-data, Fuzzy Logic, Information Mining

\section{INTRODUCTION}

The potential growth of internet, web technologies, cloud and distributed systems provides many business opportunities for the enterprises to offer a web-based services to the customers. The collaborative frameworks have already gained a huge popularity worldwide and as a result of which many online portals, blogging site, micro blogging and social media applications are booming across the globe among the users. The perceptions are built to have a large network which generates a massive and heterogeneous data consisting of html tag as semi-structured and many unstructured data which is bound to grow continuously over period of time.

Initial days challenges were to store the data and make is accessible, whereas with the maturity of cloud computing and data management schemes and statutory requirements to store the data for the longer time brought another challenge to the community of researchers to facilitates a mechanism for extracting insightful knowledge out of the reposited data. The large and unstructured data which are shared across the collaborative information network can be mined and these meaningful interpretations can be made useful in various aspects of monitoring and decision-making process that will be beneficial in many aspects.

Revised Manuscript Received on December 15, 2019.

Patil N S, Assistant Professor in the Department of Information Science, BIET, Davangere.

Dr. Kiran, Assoc. Professor in CSE Department, RNSIT, Bengaluru

Preethi B, Assistant Professor Department of Computer Science, BIET, Davangere.
It is a quite challenging task to handle the information which are into in the forms of the various tags of the codes of $\mathrm{xml}$ and html as well as in the textual unformatted structures and if this coded information adds to the existing data it makes the data enriched in many ways. This paper presents a mechanism of the scrapping technique of the data from this collaborative web information using numerical computing model so that many of the knowledge embedded inside these data can be added which reinforce the existing data strength, the reinforcement process takes place by a model of string matching using fuzzy set logic.

\section{A. Dataset Description}

The top conference related to the machine learning related domain is "Neural-Information-Processing-Systems(NIPS)" which covers various problem domain related to the subjects like

1) Cognitive-science(cs),2) deep-learning(dl),3) re-inforcement learning ( $\mathrm{rl}$ ) and 4) computer-vision( $\mathrm{cv}$ ) etc. The table I shows the description of the data sources.

Table.1 Data source description of NIPS-2015

\begin{tabular}{|c|l|l|}
\hline Sl. No & File Name & Description \\
\hline 1 & Authors.csv & $\begin{array}{l}\text { It contains id's and names for } \\
\text { each of the authors }\end{array}$ \\
\hline 2 & PaperAuthors.csv & $\begin{array}{l}\text { Linking of papers to their } \\
\text { corresponding authors. }\end{array}$ \\
\hline 3 & Papers.csv & $\begin{array}{l}\text { One row for each of the 403 } \\
\text { papers from the conference. }\end{array}$ \\
\hline 4 & Hashes.txt & Hashes \\
\hline 5 & Aceeptedpaper.htm & Accepted papers \\
\hline
\end{tabular}

\section{RELATED WORK}

The approach of data mining helps to solve many real-world problems. The typical problems space in this domain includes three basic problems 1) regression, 2) clustering and 3) classification. The ever-increasing pace of various kind of data generations brings many challenges which need to be handled includes the problem of context oriented heterogeneous data along with various kinds of uncertainty into data dues to noises and data in move. In order words a new form of data called big data in in existence which have many forms of unstructured and impure data that need to have specific approaches of models working on distributed file systems in many of the application domain including healthcare, social network, communication and the transportations system etc [1]. Taking one example of financial or banking sector, it is found that the usage of the advance digital payment systems becomes hard due to lack of proper computing infrastructures.

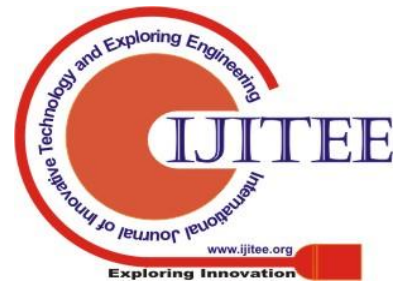


The use of micro-services of messaging is being utilized but exposed to the major security threats. The alternate scheme namely crypto currency offers some better approach but still it does not penetrate to the larger depth. The block chain-based approach on the basis of node to node communication is proposed by (Y. Hu et al., 2019), where the node graph modelling plays an important role for designing the process [2]. Ever-growing textual data cannot be analysed manually. The event detection is a problem in the unstructured text mining, where the conventional methods fails to consider the context. In the study of (G. Xu et. Al, 2019) has considered Chinses language to detect the events and applied a method of fusion of many features considering the context. The uniqueness of this approach is that the representation structure includes features like, semantic grammar, context etc which gives higher accuracy of event detection from the unstructured text [3].

There is huge popularity of the video for the information sharing. The summarization of the video content is a challenging task and modelled as a problem of subset extraction that benefits many applications and this success of these depends upon how exactly the low layer structure is considered. The principal component in changing scenario and extraction of the key frame is proposed by the (C. Dang. 2015), where they consider unstructured videos [4]. Even in the context of medical data they are not accurately annotated or labeled and found very noisy and highly unstructured with a large volume. The work by (G. Harerimana, 2019) provides insights of these data by means of deep learning approach for planning more effectively the clinical task[5]. The existence of the large data size of unstructured format is countered into drug discovery process and geometric information of the topography or geography $[6,7]$.

The use of regular expression is quite useful to handle both the semi as well as unstructured text data as there are many patterns can be interpreted by the regular expression [8]. The large volume of unstructured data can be transformed into structured data before applying any kind of the knowledge extraction and interpretation process probably using concept of ontology and learning models which leads to have insights like a) vent, 2) types of events and their relationship which is measured for its efficiency by F-measure metric[9]. (K. Liu et. Al, 2018) works on the structural format of the cancer dataset suing convolutional neural network (CNN), in contrast that CNNs are designed to handle the unstructured formats, so they modify CNN as a fully connected one to get better accuracy [10]. In the domain of social network analysis, especial unstructured settings provide a rich treasure of the information, whereas analysis location targets, activities, poses and occlusion is very cumbersome task. (Alameda-Pineda et al.,2016) suggest annotation of gestures which makes the method more effective as compare to sate of art work to get cues for the insights of the social interactions [11].

The customer data automation is achieved by (S. Agarwal, et al, 2017) using knowledge extraction, machine learning and natural language processing [12]. (W. tabib eta 1, 2018) exploits the potential of mining for the purpose of mapping and navigating task [13]. The prediction from the

Table 2 Research on the large unstructured data heterogeneous task is studied by (V. Huddar et al , 2016) using multi-view techniques on the unstructured text data[14]. There exist one another important aspect in the society that the adult context across the web is mushrooming, therefore it should be detected to be monitored or restrictions to the selected user groups. Existing filtering mechanism based on the knn, byes or n-gram or keyword match fails to get the insights from the unstructured contents from the web. This issue is handled by (F. Ali et al., 2017) with the help of technologies like svm, fuzzy based meaning [15]. The SNA using the data from the social network site can provide an effective means to identify the dynamics of the people behaviours but due to its unstructured formats of the data it is a computational cost intensive task. (A. Psyllidis, 2018), introduces method by considering spatial-temporal correlation along with topic and localization for performing clustering [16]. (M. S. Hossain et al, 2016) handled all kind of health care structured, semi structured and un-structured data of nature of big data suing svm and gaussian mixture of the model [17]. Whereas, (B. Jin et. Al, 2018), applied neural network for the heart rate prediction [18]. The knowledge ming in the field of life logging which has its applicability in the field of health care, security etc, where large unstructured data of eco-centric is analysed by (] M. Bolaños et. Al, 2017)[19]. Similarly, (L. Ponzanelli et al.,2019) has analysed video tutorials to handle the issue of search and handling the content[20].

The comprehensiveness and the most important concept from the unstructured text data require an efficient feature extraction mechanism. The authors (M. Aman et. al, 2018) has devices a tree structure of the critical feature extraction mechanism of parts of speech(pos) and uses memory less mechanism for the filtering [21]. The research for building the model of the smart grids from the unstructured and incomplete data is another area where mining approach plays an important role. An approach of automatic extraction of only power related data is being attempted by (J. Riveraet . al, 2019) using spatial models.to get accurate topology [22]. Table 2 shows some of the latest work on the large unstructured text data 


\begin{tabular}{|c|c|c|c|}
\hline Cite No. & Problem Focus & Technique used & Inferring \\
\hline E. Souza et al.,2018[23] & Text mining of Portuguese & Systematic mapping & $\begin{array}{l}\text { Newspaper and twitter as } \\
\text { corpus }\end{array}$ \\
\hline V. Menon et. al, 2019[24] & Outlier in system & PCA & $\begin{array}{lll}\text { Parameter free } & \text { outlier } \\
\text { identification } & & \end{array}$ \\
\hline M. Du et. al, 2019[25] & Unstructured log analysis & Online streaming method & $\begin{array}{lrr}\text { Dynamic } & \text { extraction } & \text { and } \\
\text { automatic } & \text { discovery } & \text { of } \\
\text { semantic } & & \end{array}$ \\
\hline F. Wang et. al, 2017[26] & Unstructured verbatim & Bi-level feature extraction & $\begin{array}{ll}\text { Worked on railway } \\
\text { maintenance dataset }\end{array}$ \\
\hline R. Kumaravel et. al, 2018[27] & $\begin{array}{ll}\text { Unstructured } & \text { documents } \\
\text { multidomain } & \text { knowledge } \\
\text { extraction } & \end{array}$ & Unified ontology modelling & $\begin{array}{l}\text { Thermal Power Plant Industry } \\
\text { and domain identification }\end{array}$ \\
\hline D. Yu et. al, 2019[28] & Social unstructured text content & $\begin{array}{l}\text { Advance online analytical } \\
\text { processing }\end{array}$ & $\begin{array}{ll}\text { Twitter hierarchical latent } \\
\text { Dirichlet allocation }\end{array}$ \\
\hline H. Guo et. al, 2016,[29] & $\begin{array}{lll}\text { Both } & \text { structured } & \text { and } \\
\text { unstructured mesh data } & \end{array}$ & Vortex extraction & $\begin{array}{l}\text { Scientists understand vortex } \\
\text { dynamics \& superconductor }\end{array}$ \\
\hline J. Leng et. al, 2017,[30] & Matching group relationship & $\begin{array}{l}\text { Semi supervised learning, graph } \\
\text { model }\end{array}$ & Prototype for social interaction \\
\hline
\end{tabular}

Many languages exist in the world apart from English, in that urdu is also spoken by large number of people. The large unstructured urdu data is analysed by (]M. Ali,2018) by a language independent classifier[31]. Agarwal eta.al, 2018 patiest data using NPL[32]. Other research domain in the large unstructured text mining includes on biomedical text using FuzzyKM[33], event log mining[34], unstructured text data from court[35], big data from health care section[36], news and event data[37]., teachers online unstructured text discussion[38], graph mining[39]. The detail survey conducted by Patil N S et al. [40]

It has been seen that almost in every walk of life the system, is producing large and unstructured data, through various method has been explored but graph mining is a technique which is explored very less.

\section{RESULTS AND DISCUSSIONS}

In order to generate potential insightful information from the unstructured collaborative web information located in a storage of big data, the study introduces a numerical modeling which enables realisation of web scrapping with cost-effective computation. Here web scrapping refers to a computational process which extract meaningful information from the collaborative web based unstructured data such as HTML tag attributes. The prime agenda of this task is to enhance the information reinforcement paradigm by providing a mining operation which establishes superior connectivity among information dissipating nodes by reducing the complexity and redundancy associated with data processing task. The entire computational operation in order to analyse the corroborative heterogeneous web data, is modelled by referencing the data source description highlighted in [Table-2: FileName, Description].

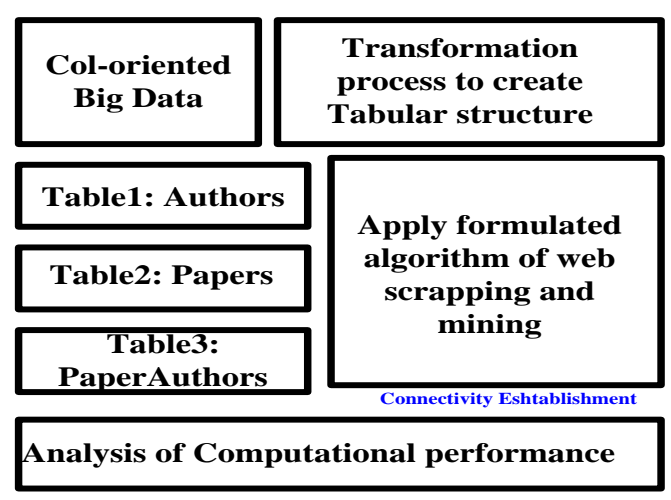

Figure 1 block-based representation of the concept

\section{a) Approach1: Numerical computation operation}

It is noted that the storage structure in Big data maintains a column-oriented data. The process initially fetches a file attribute <Authors.csv> and extracts the tabular information in the form of $\mathrm{AC}_{\mathrm{d}}$ that refers to author column-oriented data. A function $\mathrm{f}_{1}(\mathrm{x})$ performs this transformation process of creating table attributes through a specific file $\left(\mathrm{f}_{\mathrm{e}}\right)$ entities. This transformation or conversion process can be mathematically realized as follows:

$A\left([\right.$ Id, Name $] \leftarrow f_{1}\left(A C_{d}\right)$ e.q. 1

The transformation process basically generates a table namely Table: Authors $(1078 \times 2)$ which is denoted with A. The decomposition of table A revels two prime attributes such as Id and Name.

The table 3 shows an instance of table Author where the attributes are extracted from a big data column-oriented file structure in the context of larger size of heterogeneous collaborative networks. The instance of table 3 also shows a row-col oriented table structure in terms of two prime variables which are produced through applying on column-oriented data. 
Table 3 Demonstrate a row-col oriented table structure Table3: Author

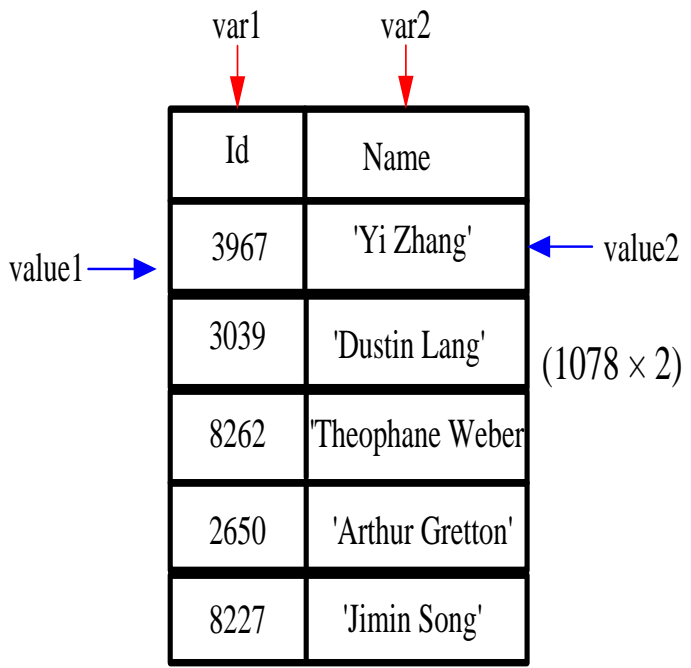

The further computational operation is numerically designed to covert the column-oriented big-data <Papers.csv> into a tabular structure of Papers. The transformation process takes places through another functional module of $\mathrm{f}_{2}(\mathrm{x})$ s.t.

$\mathrm{x} \rightarrow \quad$. Here $\quad$ refers to papers column-oriented data. The entire process is mathematically expressed as follows:

$P\left([\right.$ Id, Title, EventType, PdfName, Abstract, PaperText $] \leftarrow f_{2}\left(P C_{d}\right)$, e.q. 2

The processing of $\mathrm{PC}_{\mathrm{d}}$ data attributes result in 6-tuple oriented table Papers. An instance of the table is shown as follows:

Table 4 presents the outcome obtained after processing the mathematical expression.

\section{Tabla: Papars}

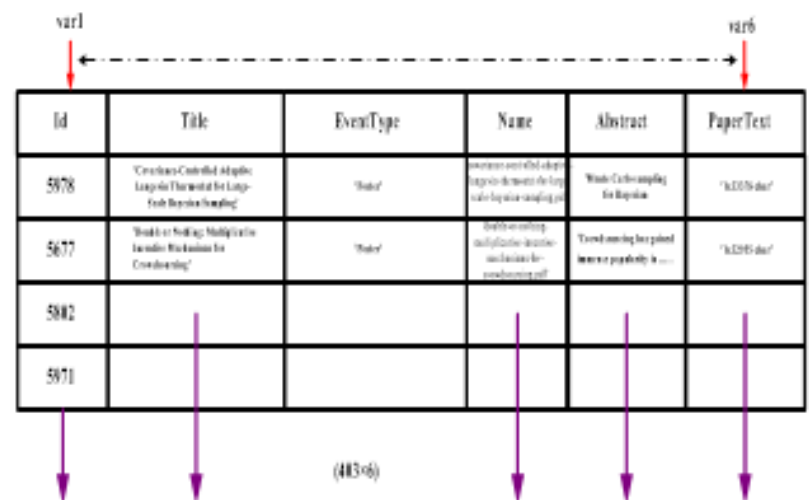

The above table 4 shows the outcome obtained after processing the mathematical expression highlighted in eq. 2 .

Another column-oriented data $P A_{c d}$ which stands for PaperAuthors column-oriented data is processed under the operational procedures attained by the functional module $\mathrm{f} 3(\mathrm{x})$. This function in return produces three prime variables such as Id, PaperId, AuthorId. The transformation process can be realized as follows:

$P A\left([I d\right.$, PaperId,AuthorId $] \leftarrow f_{3}\left(P A C_{d}\right)$ e.q. 3

The tabular structure of the attributes can be shown as follows:
Table 5 Tabular structure of the attributes

Table5: PaperAuthors

\begin{tabular}{|c|c|c|}
\hline Id & PaperId & AuthorId \\
\hline 2 & 5677 & 2649 \\
\hline 3 & 5941 & 8299 \\
\hline 4 & 5941 & 8300 \\
\hline 5 & 5941 & 575 \\
\hline 6 & 6019 & 8419 \\
\hline
\end{tabular}

$(1321 \times 3)$

The study also applies another functional module $\mathrm{f} 4(\mathrm{x})$ which reads the " $<$ html doc $>$ "file consisting of approximately 54 pages of data from a database 'datastore' considering a variable db(type). The loaded string of $\langle\mathrm{html}$ doc $\rangle$ can be displayed with number of lines as highlighted below:

\section{Table 6 Unstructured <html > number of lines} Unstructured $\langle$ html doc $>$ with number of lines 397: $\langle$ div $\rangle\langle$ div $\rangle\langle$ h3 $>$ NIPSStringLine $1</$ h 3$\rangle\langle$ p $\rangle\langle$ br $\rangle\langle/$ p $\rangle$ 398: $\langle$ i $\rangle\langle$ spanclass="StringLine $2\langle/$ span $\rangle\langle/ \mathrm{i}\rangle\langle$ br $\rangle\langle$ b $\rangle$ 399

MSR $</$ b $\rangle\langle$ br $\rangle\langle$ br $\rangle\langle$ i $\rangle<$ span class="larger-font" $>$ StringLine $3<$ span $\rangle\langle/ \mathrm{i}\rangle\langle$ br $\rangle\langle$ b $\rangle$ 400:

NICTA $\langle/$ b $\rangle\langle$ br $\rangle\langle$ br $\rangle\langle$ i $\rangle\langle$ span

class="larger-font" $>$ StringLine $4</$ span $\rangle\langle/ \mathrm{i}\rangle\langle$ br $\rangle\langle$ b $\rangle$

Further the operations load the entire text from the files and store into object $\langle$ html doc obj $>$ of size $(1 \times 1)$ with bytes of 238742. An instance of the object which hold the extracted unstructured text from the $\langle A \mathrm{p}(\mathrm{html}) /\rangle$. The further operation of the computational modeling is subjected to perform scraping operation considering the web resource from Table 4: 397 with a specific structural expression.

\section{b) Approach2: Scrapping data from the webpage}

This module is functionally designed to scrape the unstructured big data by employing the text source information analysis. It basically considers different <structural representation $\left(\mathrm{S}_{\mathrm{r}}\right)>$ metric. This procedure performs tokenization for $S r_{1}, S_{\mathrm{r} 2}$ which represents the initial and end point of a list sequence and also extracts different set of sub-strings between $S_{\mathrm{r} 1}, S_{\mathrm{r} 2}$. It also extracts different set of list items and minimize the redundancy of data by deleting the insignificant attributes.

Here $S_{\mathrm{r}}$ basically indicates the procedural execution flow to outline a sequence of characters with a defied pattern or a format. This implemented on the basis of a search algorithm procedure which also employ matching strategy. This provision the volume of text pattern for a subset of text without having exact character match.

Representation: w (ord1)? W (ord2)? (word3| word4)

Match $_{\text {index }} \leftarrow$ Match (text representation)

$\mathrm{L} \leftarrow$ match $(\mathrm{R} 1, \mathrm{R} 2, \mathrm{html})$

$\mathrm{L}_{\text {item }} \leftarrow$ match $(\mathrm{R} 3, \mathrm{R} 4, \mathrm{~L})$

$\mathrm{T} \leftarrow \operatorname{match}\left(\mathrm{R} 5, \mathrm{~L}_{\text {item }}\right)$

Here $\mathrm{L}=$ List, Litem $=$ item list and $\mathrm{T}=$ Titles

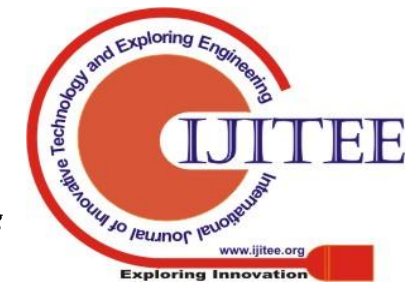


This procedure shows how the procedure performs matching operation through different types of tokenized attributes in order to explore the unique representation. The matching operation takes place in a way where the scrapped data is compared with the database table attributes. During this procedure also the computational process handles different missing gaps such as it removes the data redundancy, and also marks the missing data. The Fuzzy stricng matching operation performs a ranking which get computed on the basis of match rate evaluation. An overview of the operation of matching is shown in the following figure 2.

The following is the algorithm execution flow to illustrate the entire process with a conceptualization modeling.

Algorithm: Scrapping and Mining unstructured collaborative web data

Input: $\mathrm{U}_{\mathrm{d}}$ web (Unstructured web data)

Output: Efficient Information mining

Start

1. Initialize $P A C_{d}, A C_{d}, P C_{d}$
a. Enable $f_{1}\left(A C_{d}\right)$
b. Enable $f_{2}\left(P C_{d}\right)$
c. Enable $f_{3}\left(P A C_{d}\right)$

2. Compute the Table operation using eq. 1 to eq. 3

3. Load $\rightarrow$ object $<$ html doc obj $>/ /$ Unstructured data

4. Perform scrapping of data from web-page

a. Pattern analysis and data redundancy minimization

5. Matching of scraped data with database Table $\left(\mathrm{D}_{\mathrm{T}}\right)$

6. Activate: $\mathrm{f}_{5}(\mathrm{X})$ : Fuzzy Matching
7. Update $\rightarrow$ missing data $\mid$ Substring matching | Review Process

8. Deletion of table Activated:

a. Perform of inner joint of table through a common key attribute

b. Construct array with accumulation

c. Create directed graph with connected components

9. Visualize the connected network of authors through unstructured data graph

End

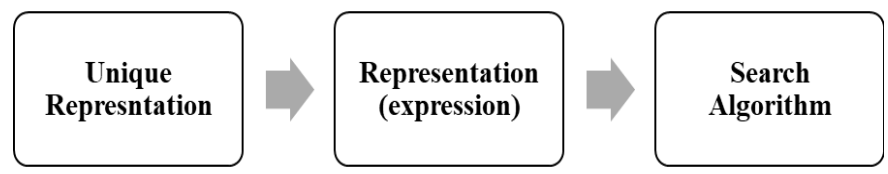

Figure 2 Process overview of string-matching operation

The above algorithm shows how the procedural modeling is designed to perform efficient connectivity establishment through web-based information mining where it also shows how the graph structure is established to find out efficient connection map vector which establishes correlation among the web-based nodes. The computational analysis shows that the procedure accomplishes to reducing the missing data by $89.3 \%$ with cost effective computation and also reduced with $35.5 \%$ during matching operation. The following figure shows the connectivity among different authors/nodes in different universities through OutDegree and InDegree computation metric.

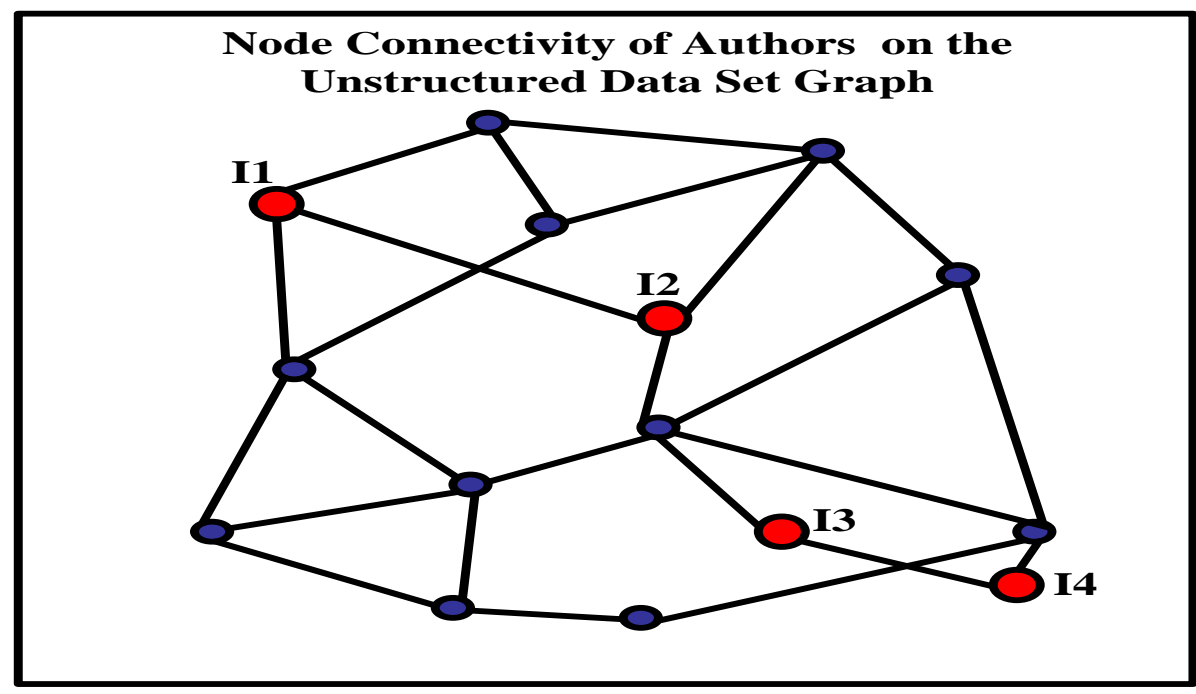

Figure 3 connectivity graph among different nodes through Fuzzy based matching algorithm

Figure 3 shows how the connectivity among different potential nodes are obtained though information mining and web scrapping operations as discussed in the methodology portion of the research study.

\section{CONCLUSION}

The study introduces a novel modeling of scrapping operation in which collecting and mining are the key-procedures to extract meaningful data from the unstructured data attributes.
The process is computationally designed with fuzzy string-matching operation which strengthens re-enforcement operation on different data attributes. The connected graph components also denote the correlation metric among potential nodes though outdegree and indegree computation. 
The numerical computational analysis clearly shows that the formulated system can handle by minimizing the data redundancy $89.3 \%$ and which is found quite lesser in the study of M. Du et. al, 2019[25]. The study also found that the formulated system also outperforms the model of M. Du et. al, 2019[25] when complexity is concerned. Thereby the formulated approach of web scrapping poses futuristic scope of this study into many application areas in the context of Industry 4.0 and social media analysis.

\section{REFERENCES}

1. W. Ding et al., "IEEE Access Special Section Editorial: Data Mining and Granular Computing in Big Data and Knowledge Processing," in IEEE Access, vol. 7, pp. 47682-47686, 2019.

2. Y. Hu et al., "A Delay-Tolerant Payment Scheme Based on the Ethereum Blockchain," in IEEE Access, vol. 7, pp. 33159-33172, 2019.

3. G. Xu, Y. Meng, X. Zhou, Z. Yu, X. Wu and L. Zhang, "Chinese Event Detection Based on Multi-Feature Fusion and BiLSTM," in IEEE Access, vol. 7, pp. 134992-135004, 2019.

4. C. Dang and H. Radha, "RPCA-KFE: Key Frame Extraction for Video Using Robust Principal Component Analysis," in IEEE Transactions on Image Processing, vol. 24, no. 11, pp. 3742-3753, Nov. 2015. doi: 10.1109/TIP.2015.2445572

5. G. Harerimana, J. W. Kim, H. Yoo and B. Jang, "Deep Learning for Electronic Health Records Analytics," in IEEE Access, vol. 7, pp. 101245-101259, 2019.

6. R. L. Martin et al., "Hybrid natural language processing for high-performance patent and literature mining in IBM Watson for Drug Discovery," in IBM Journal of Research and Development, vol. 62, no. 6, pp. 8:1-8:12, 1 Nov.-Dec. 2018.

7. J. Sreevalsan-Nair, A. Jindal and B. Kumari, "Contour Extraction in Geometric Descriptors and Visual Analytics," in IEEE Journal of Selected Topics in Applied Earth Observations and Remote Sensing, vol. 11, no. 7, pp. 2320-2335, July 2018

8. A. Bartoli, A. De Lorenzo, E. Medvet and F. Tarlao, "Inference of Regular Expressions for Text Extraction from Examples," in IEEE Transactions on Knowledge and Data Engineering, vol. 28, no. 5, pp. 1217-1230, 1 May 2016.

9. J. A. Reyes and A. Montes, "Learning Discourse Relations from News Reports: An Event-driven Approach," in IEEE Latin America Transactions, vol. 14, no. 1, pp. 356-363, Jan. 2016.

10. K. Liu, G. Kang, N. Zhang and B. Hou, "Breast Cancer Classification in IEEE Access, vol. 6, pp. 23722-23732, 2018.

11. [11] X. Alameda-Pineda et al., "SALSA: A Novel Dataset for Multimodal Group Behavior Analysis," in IEEE Transactions on Pattern Analysis and Machine Intelligence, vol. 38, no. 8, pp. 1707-1720, 1 Aug. 2016.

12. S. Agarwal, V. Aggarwal, A. R. Akula, G. B. Dasgupta and G. Sridhara, "Automatic problem extraction and analysis from unstructured text in IT tickets," in IBM Journal of Research and Development, vol. 61, no. 1, pp. 4:41-4:52, 1 Jan.-Feb. 2017.

13. W. Tabib, C. O'Meadhra and N. Michael, "On-Manifold GMM Registration," in IEEE Robotics and Automation Letters, vol. 3, no. 4, pp. 3805-3812, Oct. 2018.

14. V. Huddar, B. K. Desiraju, V. Rajan, S. Bhattacharya, S. Roy and C. K. Reddy, $\quad$ Predicting Complications in Critical Care Using Heterogeneous Clinical Data," in IEEE Access, vol. 4, pp. 7988-8001, 2016.

15. F. Ali et al., "A Fuzzy Ontology and SVM-Based Web Content Classification System," in IEEE Access, vol. 5, pp. 25781-25797, 2017.

16. A. Psyllidis, J. Yang and A. Bozzon, "Regionalization of Social Interactions and Points-of-Interest Location Prediction With Geosocial Data," in IEEE Access, vol. 6, pp. 34334-34353, 2018.

17. M. S. Hossain and G. Muhammad, "Healthcare Big Data Voice Pathology Assessment Framework," in IEEE Access, vol. 4, pp. 7806-7815, 2016.3

18. B. Jin, C. Che, Z. Liu, S. Zhang, X. Yin and X. Wei, "Predicting the Risk of Heart Failure With EHR Sequential Data Modeling," in IEEE Access, vol. 6, pp. 9256-9261, 2018.

19. M. Bolaños, M. Dimiccoli and P. Radeva, "Toward Storytelling From Visual Lifelogging: An Overview," in IEEE Transactions on Human-Machine Systems, vol. 47, no. 1, pp. 77-90, Feb. 2017. Buildings in Airborne LiDAR Point Clouds Using Multiscale Local Based on Fully-Connected Layer First Convolutional Neural Networks,"

20. L. Ponzanelli et al., "Automatic Identification and Classification of Software Development Video Tutorial Fragments," in IEEE Transactions on Software Engineering, vol. 45, no. 5, pp. 464-488, 1 May 2019.

21. M. Aman, A. b. Md Said, S. J. Abdul Kadir and I. Ullah, "Key Concept Identification: A Sentence Parse Tree-Based Technique for Candidate Feature Extraction From Unstructured Texts," in IEEE Access, vol. 6, pp. 60403-60413, 2018

22. J. Rivera, P. Nasirifard, J. Leimhofer and H. Jacobsen, "Automatic Generation of Real Power Transmission Grid Models From Crowdsourced Data," in IEEE Transactions on Smart Grid, vol. 10, no. 5, pp. 5436-5448, Sept. 2019

23. E. Souza et al., "Characterising text mining: a systematic mapping review of the Portuguese language," in IET Software, vol. 12, no. 2, pp. 49-75, 42018.

24. V. Menon and S. Kalyani, "Structured and Unstructured Outlier Identification for Robust PCA: A Fast Parameter Free Algorithm," in IEEE Transactions on Signal Processing, vol. 67, no. 9, pp. 2439-2452, 1 May1, 2019.

25. M. Du and F. Li, "Spell: Online Streaming Parsing of Large Unstructured System Logs," in IEEE Transactions on Knowledge and Data Engineering, vol. 31, no. 11, pp. 2213-2227, 1 Nov. 2019.

26. F. Wang, T. Xu, T. Tang, M. Zhou and H. Wang, "Bilevel Feature Extraction-Based Text Mining for Fault Diagnosis of Railway Systems," in IEEE Transactions on Intelligent Transportation Systems, vol. 18, no. 1, pp. 49-58, Jan. 2017.

27. [27] R. Kumaravel, S. Selvaraj and C. Mala, "A Multidomain Layered Approach in Development of Industrial Ontology to Support Domain Identification for Unstructured Text," in IEEE Transactions on Industrial Informatics, vol. 14, no. 9, pp. 4033-4044, Sept. 2018.

28. D. Yu, D. Xu, D. Wang and Z. Ni, "Hierarchical Topic Modeling of Twitter Data for Online Analytical Processing," in IEEE Access, vol. 7, pp. 12373-12385, 2019.

29. H. Guo, C. L. Phillips, T. Peterka, D. Karpeyev and A. Glatz, "Extracting, Tracking, and Visualizing Magnetic Flux Vortices in 3D Complex-Valued Superconductor Simulation Data," in IEEE Transactions on Visualization and Computer Graphics, vol. 22, no. 1, pp. 827-836, 31 Jan. 2016.

30. J. Leng and P. Jiang, "Mining and Matching Relationships From Interaction Contexts in a Social Manufacturing Paradigm," in IEEE Transactions on Systems, Man, and Cybernetics: Systems, vol. 47, no. 2, pp. 276-288, Feb. 2017.

31. ]M. Ali, S. Khalid and M. H. Aslam, "Pattern Based Comprehensive Urdu Stemmer and Short Text Classification," in IEEE Access, vol. 6, pp. 7374-7389, 2018.

32. Agarwal, C. Baechle, R. Behara and X. Zhu, "A Natural Language Processing Framework for Assessing Hospital Readmissions for Patients With COPD," in IEEE Journal of Biomedical and Health Informatics, vol. 22, no. 2, pp. 588-596, March 2018.

33. J. Rashid et al., "Topic Modeling Technique for Text Mining Over Biomedical Text Corpora Through Hybrid Inverse Documents Frequency and Fuzzy K-Means Clustering," in IEEE Access, vol. 7, pp. 146070-146080, 2019.

34. W. Song, F. Chen, H. Jacobsen, X. Xia, C. Ye and X. Ma, "Scientific Workflow Mining in Clouds," in IEEE Transactions on Parallel and Distributed Systems, vol. 28, no. 10, pp. 2979-2992, 1 Oct. 2017. doi: 10.1109/TPDS.2017.2696942

35. A. Iftikhar, S. W. Ul Qounain Jaffry and M. K. Malik, "Information Mining From Criminal Judgments of Lahore High Court," in IEEE Access, vol. 7, pp. 59539-59547, 2019.

36. J. Peral, A. Ferrández, D. Gil, R. Muñoz-Terol and H. Mora, "An Ontology-Oriented Architecture for Dealing With Heterogeneous Data Applied to Telemedicine Systems," in IEEE Access, vol. 6, pp. 41118-41138, 2018.

37. J. Wan, P. Zheng, H. Si, N. N. Xiong, W. Zhang and A. V. Vasilakos, "An Artificial Intelligence Driven Multi-Feature Extraction Scheme for Big Data Detection," in IEEE Access, vol. 7, pp. 80122-80132, 2019 Q. Liu, S. Zhang, Q. Wang and W. Chen, "Mining Online Discussion Data for Understanding Teachers Reflective Thinking," in IEEE Transactions on Learning Technologies, vol. 11, no. 2, pp. 243-254, 1 April-June 2018.

38. J. Xuan, J. Lu, G. Zhang and X. Luo, "Topic Model for Graph Mining," in IEEE Transactions on Cybernetics, vol. 45, no. 12, pp. 2792-2803, Dec. 2015. 
39. Patil, N. S., P. Kiran, N. P. Kiran, and Naresh Patel KM. "A Survey on Graph Database Management Techniques for Huge Unstructured Data." International Journal of Electrical and Computer Engineering 8, no. 2 (2018): 1140.

\section{AUTHOR'S PROFILE}

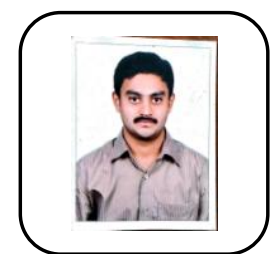

Patil N S, working as Assistant Professor in the Department of Information Science, BIET, Davangere. Myteaching experience is 9yrs with an area of interest Database management system, Data-mining, Graph Data-mining and Big Data.

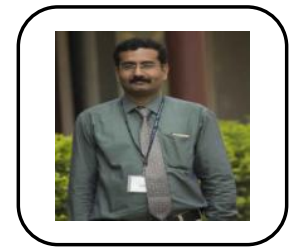

Dr. Kiran P, BE, M.Tech, Ph.D, working as Assoc. Professor in CSE Department, RNSIT, Bengaluru from past 18 years. His research interests include Cryptography, Big Data Analytics, Data Mining, Privacy Preserving Data Mining and Privacy Preserving Data Publishing. .

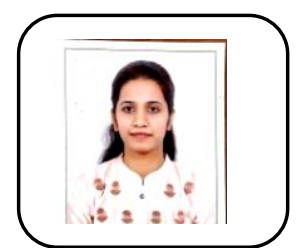

Preethi B, working as Assistant Professor Department of Computer Science, BIET, Davangere. Her teaching experience is 5yrs with an area of interest Database management system, Data-mining, Computer Graphics, Cloud computing 